\title{
Vision-Related Quality of Life After Vitrectomy: Cross-Sectional Study from Jordan
}

\author{
Mohammed Abu-Ameerh \\ Ayat Alni'mat \\ Mo'ath AIShawabkeh \\ Saif Aldeen AlRyalat (iD \\ Ophthalmology Department, Jordan \\ University Hospital, University of Jordan, \\ Amman, Jordan
}

Background: In the era of patient-centered medical care, using objective clinical measures to assess patient-centered outcomes in all aspects became a necessity, and pars plana vitrectomy (PPV) is a major ophthalmic surgical procedure used for the treatment of vitreoretinal disorders aiming to improve their vision-related quality of life.

Purpose: To study the impact of PPV on visual quality of life by assessing its effect on common daily activities, to assess its association with various factors, and to explore the relations between these factors and postoperative visual function.

Methods: Vision-related quality of life for 87 patients who underwent PPV was assessed using the 25-item National Eye Institute Visual Function Questionnaire (NEI VFQ-25) by phone call interviews. Variables assessed include age, gender, indication of surgery, duration since surgery, preoperative best-corrected visual acuity (BCVA), postoperative BCVA, fellow eye BCVA, medical history and lens status.

Results: The factors significantly affecting the total score were postoperative visual acuity and fellow eye VA. Upon analyzing the effect of the indication on total score, a significant difference was found with the highest being for those who had dropped lens as the indication for surgery and the lowest was for those with tractional retinal detachment (TRDs) and inflammatory indications. Subscale analysis and visual acuity improvement were also varying between indications.

Conclusion: VRQOL significantly improves after PPV, the improvement is variable with different indications, being the greatest for those with dropped lens and the least for TRDs and endophthalmitis, with postoperative VA being the most important factor affecting the VRQOL score.

Keywords: pars plana vitrectomy, quality of life, vision, Jordan, ophthalmology

\section{Introduction}

In the era of patient-centered medical care, using objective clinical measures alone for visual acuity (VA) seems to fail to account for a patient's overall visual function; thus, functional assessment and health-related quality of life surveys are more widely adopted as a more comprehensive and effective measure of patient vision-related quality of life (VR-QOL). ${ }^{1,2}$

The importance of surgical treatment for vitreoretinal disorders is usually clear to an individual patient and to his/her ophthalmologist, and prior studies have reported the influence of vitrectomy on vision VR-QOL in patients with proliferative diabetic retinopathy $(\mathrm{PDR})$, retinal detachment $(\mathrm{RD})$, macular hole $(\mathrm{MH})$, epiretinal membrane (ERM), and age-related macular degeneration (AMD). Such studies have demonstrated that vitrectomy can improve patients' visual function as well as VRQOL. ${ }^{3}$
Correspondence: Mo'ath AlShawabkeh Ophthalmology Department, Jordan University Hospital, University of Jordan, Amman, I 1942, Jordan

Email moathalshawabkeh12@gmail.com 
However, all these studies were done in developed countries like Japan and USA and none was conducted here in our country, the Hashemite Kingdom of Jordan.

Jordan is a developing country with different overall health statuses, disease prevalence and socioeconomic characteristics of its population; therefore, we aimed in this paper to study whether all these differences would be associated with variation in VRQOL scores as compared with those in developed countries in addition to studying the association between different indications for surgery and the postoperative VRQOL.

\section{Study Design}

This retrospective cross-sectional study was conducted between December 2019 and June 2020 at Jordan University Hospital, which is a tertiary hospital located in Amman, the capital of the Hashemite Kingdom of Jordan. Eighty-seven patients with a history of undergoing pars plana vitrectomy in our Ophthalmology Department were surveyed.

The aim of the study was explained to the subjects before starting the questionnaire, and they were asked whether they would accept to participate.

Informed Consent was obtained from all study subjects, the study was approved by the Institutional Review Board for Human Research at Jordan University Hospital and all principles outlined in the Declaration of Helsinki were followed in carrying out this research.

\section{Materials and Methods}

The clinical records of patients who underwent pars plana vitrectomy in the ophthalmology department at Jordan University Hospital between April 2015 and November 2019 were reviewed.

We included seventy eighty patients with a history of undergoing PPV for one or both eyes in the last five years, subjects included were of any age, both genders, with various indications, and had their surgery done in the same institute, same settings, and by the same consultant vitreoretinal surgeon (Dr. MAA).

All vitrectomy procedures were performed with the Dutch Ophthalmic Research Center (D.O.R.C. Associate ${ }^{\circledR}$ Dutch Ophthalmic) 20-gauge vitrectomy system.

We excluded patients who had scleral buckle prior to PPV and those who underwent redo surgery. Also, patients with other ocular diseases that can be a confounding factor in compromising patient visual function, such as corneal ectatic diseases, dystrophies or scars, glaucoma, age- related macular degeneration, retinal dystrophies, and congenital ocular anomalies, were excluded.

\section{Variables}

We reviewed subject's hospital electronic health records to retrieve following variables: age, gender, indication for surgery, duration since surgery, preoperative best-corrected visual acuity (BCVA), postoperative BCVA, fellow eye BCVA all using illiterates $\mathrm{E}$ chart, past medical history of diabetes (DM) and hypertension (HTN) and lens status, in case of bilateral surgery, we took in the study the last eye operated.

We used the Arabic version of the National Eye Institute visual function questionnaire (NEI-VFQ 25) (Supplementary 1) to assess patient's postoperative quality of life via phone call interviews, Arabic version of NEIVFQ-25 is a valid and reliable instrument for addressing and assessing VRQOL for Arabic-speaking patients ${ }^{4}$ the use of phone interview was chosen to avoid medical interactions or bias and to gather information as perceived by the patients him/herself and although the patients' medical doctors have conducted these interviews, they were blind for their medical records, diagnosis, and indication for surgery in order to prevent any bias.

The NEI VFQ-25 (Supplementary 2) consists of 25 questions that assess 12 subscales grouped into 3 main categories. The first one is general health (1 question). The second one which addresses the quality of vision and encompasses 5 subscales: the general vision (1 question), difficulty with distance tasks (3 questions), difficulty with near tasks (3 questions), peripheral vision (1 question), and color vision (1 question) subscales. The third category includes 6 subscales assessing visual function and quality of life: dependency (3 questions), role limitations (2 questions), mental health (4 questions), social function (2 questions), driving (2 questions), and ocular pain (2 questions). ${ }^{1}$

The questionnaire required 25 to 30 minutes to be completed, reasons for using this questionnaire include the patient-driven basis of its content; also, the value of comparing the relative burden of one condition with another on the same scale; the multifactorial nature of these questionnaire subscales, which are designed to recognize the impact of visual problems on physical functioning, emotional health, and social functioning; and finally the multi-condition evaluation of the reliability and validity of the NEI VFQ- $25 .^{5}$ 
We categorized indications into: Vitreous Hemorrhage (VH) that is persistent non-clearing for more than 3 months with at least one intravitreal injection provided that traction has been ruled out using B-scan ultrasonography, Rhegmatogenous Retinal Detachment (RRD) due to horseshoe tear with posterior vitreous detachment (PVD), Tractional Retinal Detachment (TRD) which threatens or involves the macula, Macular hole with deteriorating of visual acuity (VA) on follow up or with VA of less than 0.2 $(20 / 100)$ at the time of presentation, Epiretinal membrane defined as a translucent membrane with macular thickening involving the center of the macula, with or without distortion and wrinkling of the inner retinal surface on slit-lamp examination and OCT; with vitrectomy being indicated for patients with significant metamorphopsia, Dropped lens including dropped nucleus, lens particle or intraocular lens (IOL), and inflammatory including uveitis and endophthalmitis which did not improve by conservative measures.

We converted counting fingers, hand motion, light perception, and no light perception into $0.014,0.005,0.0016$, and 0.0013 , respectively. ${ }^{6}$

\section{Statistical Analysis}

We used SPSS version 21.0 (Chicago, USA) in our analysis. We used the mean ( \pm standard deviation) to describe continuous variables (eg age). We used count (frequency) to describe other nominal variables (eg gender).

We adopted a model-building strategy to analyze the factors affecting the total score, where only factors with a significant threshold higher than 0.1 , as found by chi-square and one-way ANOVA or $t$-test were entered into the linear regression analysis. Furthermore, as the indication of surgery may affect the score, regardless of other factors, we used oneway ANOVA to analyze the difference in total score and change in visual acuity between different indications, and we used Tukey post-hoc test. All underlying assumptions were met unless otherwise indicated. We adopted a p-value of 0.05 as a significant threshold.

\section{Results}

A total of 87 patients were included in this study, with a mean age of $57.49( \pm 12.93)$ years. They were 53 $(60.9 \%)$ men and 34 (39.1\%) women. Sixty-six (75.9\%) of them were diabetic and $51(58.6 \%)$ were hypertensive. Thirty-seven (42.5\%) were for right eyes, 27 (31.0\%) were for left eyes and $23(26.4 \%)$ had bilateral surgery. The mean duration between surgery and the study was $25.39( \pm 16.48)$ months. Most of our patients have posterior chamber intraocular lens (IOL), and there were two patients with anterior chamber IOL and one aphakic patient. Fifty-one (58.6\%) had better VA post-op, 22 (25.3\%) had worsened VA, and $14(16.1 \%)$ had the same VA. Table 1 details the characteristics of the included sample.

In the regression analysis, the model overall significance was $<0.001$, with an adjusted $\mathrm{R}$ square of 0.317 . The only factors that were significantly affecting the total score were post-op visual acuity ( $\mathrm{p}=0.001, \mathrm{~B}=27.58$, and beta $=0.319)$, and visual acuity of the other eye $(\mathrm{p}<0.001, \mathrm{~B}=28.20$, and beta $=0.411$ ). Meaning that a change of one unit in post-op visual acuity would result in an increase in total score by 27.58 , while a change of one unit in other eye visual acuity would result in an increase in total score by 28.20

Upon analyzing the statistical relationship between the indication for surgery and VRQOL composite score, we found a significant difference in the score with different indications $(p=0.008)$. In post-hoc analysis, the lowest scores were for patients with TRD and inflammatory indications, and the highest score was for those with dropped lens (Table 2).

On analyzing the visual acuity change after vitrectomy between different indications, a significant difference was found $(\mathrm{p}=0.01)$, with dropped lens and $\mathrm{VH}$ indications carrying the best prognosis in terms of postoperative visual acuity improvement from preoperative baseline VA as shown in (Table 3).

We studied the relation between the different indications and the NEI-VFQ25 subscales as demonstrated in Table S1 (Supplementary File):

General health subscale score was highest for those with dropped lens and macular hole followed by RRD, and lowest for those with TRD, and VH.

General vision score was highest for those with macular hole and dropped lens, least for those with ERM indication followed by inflammatory indication than TRD.

For the ocular pain domain, there was no significant difference between indications, yet inflammatory indication had the most pain.

For near activities, distance vision and social functioning domains, the dropped lens had the highest score, the lowest score was for TRDs and inflammatory indications.

Mental health and role difficulty subscale scores were the highest in the dropped lens and macular hole groups and the lowest in TRDs and inflammatory groups.

For dependency domain, the highest score belongs to the dropped lens and macular hole patients, and the lowest for TRDs and inflammatory groups. 
Table I Demographic Characteristics of the Sample

\begin{tabular}{|c|c|c|c|c|c|}
\hline & & Mean & Standard Deviation & Count & Column N \% \\
\hline \multicolumn{2}{|l|}{ Age } & 57 & 13 & & \\
\hline \multicolumn{2}{|c|}{ Duration since surgery } & 25.39 & 16.48 & & \\
\hline \multirow[t]{7}{*}{ Indication } & $\mathrm{VH}$ & & & 31 & $35.6 \%$ \\
\hline & RRD & & & 20 & $23.0 \%$ \\
\hline & TRD & & & 21 & $24.1 \%$ \\
\hline & Macular hole & & & 2 & $2.3 \%$ \\
\hline & Dropped IOL & & & 6 & $6.9 \%$ \\
\hline & Inflammatory & & & 4 & $4.6 \%$ \\
\hline & ERM & & & 3 & $3.4 \%$ \\
\hline \multicolumn{2}{|l|}{ Preop VA } & 0.15 & 0.22 & & \\
\hline \multicolumn{2}{|l|}{ Postop VA } & 0.24 & 0.27 & & \\
\hline \multicolumn{2}{|l|}{ VA change } & 0.09 & 0.34 & & \\
\hline \multirow[t]{3}{*}{ Improved VA } & Improved & & & 51 & $58.6 \%$ \\
\hline & Same & & & 14 & $16.1 \%$ \\
\hline & Worsened & & & 22 & $25.3 \%$ \\
\hline \multicolumn{2}{|c|}{ VA another eye } & 0.48 & 0.35 & & \\
\hline \multirow[t]{3}{*}{ Lens } & $\mathrm{PCIOL}$ & & & 84 & $96.6 \%$ \\
\hline & Aphakic & & & 1 & $1.1 \%$ \\
\hline & $\mathrm{AClOL}$ & & & 2 & $2.3 \%$ \\
\hline \multirow[t]{2}{*}{ DM } & Yes & & & 66 & $75.9 \%$ \\
\hline & No & & & 21 & $24.1 \%$ \\
\hline \multirow[t]{2}{*}{ HTN } & Yes & & & 51 & $58.6 \%$ \\
\hline & No & & & 36 & $41.4 \%$ \\
\hline
\end{tabular}

Abbreviations: $\mathrm{AClOL}$, anterior chamber intraocular lens; $\mathrm{PCIOL}$, posterior chamber intraocular lens.

Table 2 Indications for PPV and Total Score

\begin{tabular}{|l|l|l|l|l|l|}
\hline & The Number of Patients & Mean & Std. Deviation & \multicolumn{2}{|l|}{ 95\% Confidence Interval for Mean } \\
\cline { 4 - 7 } & & & & Lower Bound & Upper Bound \\
\hline VH & 31 & 73.1645 & 21.85241 & 65.1490 & 81.1800 \\
\hline RRD & 20 & 80.4700 & 15.07110 & 73.4165 & 87.5235 \\
\hline TRD & 21 & 58.2048 & 28.90755 & 45.0462 & 71.3633 \\
\hline Macular hole & 2 & 88.8500 & 15.76848 & -52.8242 & 230.5242 \\
\hline Dropped lens & 6 & 92.4000 & 2.74153 & 89.5229 & 95.2771 \\
\hline Inflammatory & 4 & 60.6750 & 20.94029 & 27.3543 & 93.9957 \\
\hline ERM & 3 & 73.6667 & 22.90728 & 16.7618 & 130.5715 \\
\hline Total & 87 & 72.3632 & 23.39092 & 67.3779 & 77.3485 \\
\hline
\end{tabular}


Table 3 Mean Difference in Visual Acuity and Indication for PPV

\begin{tabular}{|l|l|l|l|l|l|}
\hline & The Number of Patients & Mean Change in VA & Std. Deviation & \multicolumn{2}{l|}{ 95\% Confidence Interval for Mean } \\
\cline { 3 - 6 } & & & & Lower Bound & Upper Bound \\
\hline VH & 31 & 0.1986 & 0.25311 & 0.1057 & 0.2914 \\
\hline RRD & 20 & 0.0094 & 0.34936 & -.1541 & 0.1729 \\
\hline TRD & 21 & 0.0533 & 0.30918 & -0.0875 & 00.1940 \\
\hline Macular hole & 2 & -0.1930 & 0.13152 & -10.3747 & 0.9887 \\
\hline Dropped IOL & 6 & 0.4240 & 0.40153 & 0.0026 & 0.8454 \\
\hline Inflammatory & 4 & -0.3974 & 0.35054 & -0.9551 & 0.1604 \\
\hline ERM & 3 & 0.0667 & 0.11547 & -0.2202 & 0.3535 \\
\hline Total & 87 & 0.0946 & 0.33540 & 0.0231 & 0.1661 \\
\hline
\end{tabular}

Driving subscale score was found to be the highest in the dropped lens and the lowest in TRD.

Color vision highest score was for macular hole and dropped lens and the lowest score was for inflammatory and TRDs.

Peripheral vision highest score was for the dropped lens, the lowest for TRDs; however, unlike other subscales, the inflammatory group score was the 2 nd highest.

\section{Discussion}

Increasing research efforts and funding has been directed toward the assessment of patient-centered outcomes, most importantly quality of life. ${ }^{7}$

In our study, we assessed the postoperative visionrelated quality of life using NEI-VFQ 25 for 87 patients who underwent PPV in Jordan University Hospital for different indications for surgery. The questionnaire focuses on the influence of visual disability and visual symptoms on generic health domains, such as emotional health and social functioning, in addition to task-related domains specified for daily visual functioning. It consists of 25 vision-targeted questions representing 11 vision-related subscales, with an additional, single-item, general health status question. The NEI-VFQ-25 assess the following vision-targeted subscales: binocular vision rating, difficulty with near activities, difficulty with distance vision, social functioning limitations, role limitations due to vision, dependency on others, mental health disturbances due to vision, driving difficulties, limitations of peripheral and color vision, and ocular pain. ${ }^{8}$
Many previous studies had shown evidence of improvement in VR-QOL after ophthalmic surgeries, such as cataract, photorefractive excimer laser keratectomy, laser in situ keratomileuses and vitrectomy for PDR, ERM, MH, and age-related macular degeneration ${ }^{3}$ this improvement was evident even with good vision in the fellow eye as was demonstrated by Hirneiß $\mathrm{C}$ while studying the outcome of PPV for macular hole patients. ${ }^{2}$

However, this improvement was widely variable and yet to be investigated in the era of evidence-based clinical practice; in our study, where we analyzed the relationship between the indication for PPV and VQOL composite score, we found that the highest score belongs to those who had dropped lens as the indication for surgery and the lowest was for those with TRDs and inflammatory indications.

The highest score for dropped lens could be expected from several studies showing that pars plana vitrectomy is an effective and safe method of removing retained lens fragments after phacoemulsification ${ }^{9}$ and the fact that visual acuity generally improves in most patients postoperatively after $\mathrm{PPV}^{10}$ with most eyes achieving final visual acuity of 20/40 or better after the surgery. ${ }^{11-14}$

While the lowest score was for the inflammatory group in which most of the cases in our study were endophthalmitis patients, this has been demonstrated by other studies, such as Ng JQ who stated that despite dramatic changes that have occurred in the management of postoperative endophthalmitis during the past decade, visual outcomes did not improve much and it remained generally poor. ${ }^{15}$ 
The same applies to TRDs where several studies demonstrated that visual outcomes after PPV remained limited despite the improvement in the anatomical success rates $^{16}$ with the percentage of eyes having final VA of 20/ 100 or better not exceeding $40 \%$, and no more than $80 \%$ have VA of $5 / 200$ or better. ${ }^{17-22}$

As determined by this study, the only statistically significant factor affecting the total score being the postoperative visual acuity, this is consistent with Okamoto F who stated that after vitreoretinal surgery, the BCVAs showed significant correlation with the postoperative VFQ-25 composite score in PDR patients, ${ }^{3}$ this also applies to other ocular conditions as found by Cahill MT who stated that the most important NEI VFQ-25 subscales directly related to VA were the general vision, distance activities, and near tasks subscales, indicating that patients with poor overall vision had more difficulties in most vision-dependent daily activities. ${ }^{1}$

In terms of VA improvement after surgery in this research, the biggest improvement was for $\mathrm{VH}$ and dropped lens. However, in RRD, the preoperative VA was relatively high, and thus surgery-induced improvement in visual function and acuity remained small, this explains the good VRQL score despite minimal or no improvement in postoperative VA. The visual outcome after PPV for VH was widely studied, generally in patients with vitreous hemorrhage, one can expect significant visual improvement in around half of the cases with good visual outcome provided that retina is not detached. $^{23}$

This study results are consistent with previous studies comparing PPV outcome in VH versus TRD patients as a complication of diabetes, which showed poorer outcomes for TRDs. ${ }^{23}$

This study subscale analysis based on NEI VFQ25 twelve domains showed that patients with dropped lens generally exhibit the highest score in all subscale domains with inflammatory conditions and TRDs having the worst score except for peripheral vision subscale in which inflammatory groups had the second-highest score after dropped lens. Patients with TRDs tend to have poor overall health with a high coincidence of metabolic syndrome and higher mortality rate, ${ }^{16}$ this can explain the low general health subscale score for this group, also, the poor visual outcome of PPV for this condition ${ }^{24}$ generally explains the decreased other subscale scores.

Many studies have focused on mental health for ophthalmic conditions and after ophthalmic interventions, patients with varying degrees of visual impairment generally have increased risk of depression, and impaired visual function is considered an independent predictor of suicide in the elderly ${ }^{25}$ Patients with various eye diseases, including age-related macular degeneration, glaucoma, retinitis pigmentosa, tend to have decreased quality of life and various degrees of depression. ${ }^{26}$

Another study ${ }^{27}$ discussing the impact of ophthalmic interventions, such as cataract surgery on mental health, showed that within 1 year of treatment, change in visual function was accompanied by significant changes, in the same direction, in vision-related quality of life including mental well-being, and life satisfaction.

This was also found in another study done by Ishii K, which showed that the change in best-corrected visual acuity after surgery significantly correlated with the change in NEI VFQ-25 score and with the change in mini-mental state exam (MMSE) score. ${ }^{28}$

In this study, mental health score was high for the dropped lens $(83.3 \%)$ and macular hole $(87.5 \%)$, low in TRDs (42.2\%) and inflammatory (34.4\%) and intermediate for RRD (69.0\%), ERM (68.7\%), and VH (62.3\%).

This paper has some limitations: first, as we study the impact of vitrectomy on quality of life, it is well known from previous studies that vitrectomy has a moderate to large size effect on quality of life. ${ }^{6}$ Based on Pearson's model for sample size estimation, and to achieve a power of $80 \%$, a sample size between 80 and 190 is required. ${ }^{29}$ While our sample size is still in the lower limit of the required sample size, we believe further research should concentrate on larger population samples since individuals may respond to questions in an overly positive or overly negative manner, depending on personality traits, motivations, or incentives. Moreover, analysis of visual function impairment and VRQOL assessed data only on central visual acuity. Clearly, visual impairment has many aspects including contrast sensitivity, metamorphopsia, peripheral field, stereo acuity, and others.

\section{Conclusion}

VRQOL significantly improves after PPV, the improvement is variable with different indications, being the greatest for those with dropped lens and the least for TRDs and endophthalmitis, with postoperative VA being the most important factor affecting the VRQOL score, our results were consistent with previous studies in ophthalmology centers in other regions.

This supports the established importance of tight glycemic control in order to prevent advanced diabetic eye disease, such as TRDs, the need for further surgical improvement and earlier medical management for diabetic retinopathy and to emphasize on the importance of adherence to the sterile techniques 
and guidelines for ophthalmic interventions to reduce the risk of endophthalmitis.

\section{Data Sharing Statement}

The datasets supporting the conclusions of this article are available upon request to the corresponding authors. Due to data protection restrictions and participant confidentiality, we do not make participants' data publicly available.

\section{Consent to Participate}

Participation in the study was voluntary, and individuals were free to withdraw or stop the interview at any time. Informed consent was obtained for all individual participants included in the study.

\section{Acknowledgments}

The authors would like to thank the National eye Institute (NEI) for providing us with their visual function questionnaire 25 that was used in this study and for their support for health and vision researches and programs.

\section{Disclosure}

The authors declare that they have no conflicts of interest.

\section{References}

1. Cahill MT, Banks AD, Stinnett SS, Toth CA. Vision-related quality of life in patients with bilateral severe age-related macular degeneration. Ophthalmology. 2005;112(1):152-158. doi: $10.1016 / \mathrm{j}$ ophtha.2004.06.036

2. Hirneiß C, Neubauer AS, Gass CA, et al. Visual quality of life after macular hole surgery: outcome and predictive factors. $\mathrm{Br}$ J Ophthalmol. 2007;91(4):481-484. doi:10.1136/bjo.2006.102376

3. Okamoto F, Okamoto Y, Fukuda S, Hiraoka T, Oshika T. Visionrelated quality of life and visual function after vitrectomy for various vitreoretinal disorders. Invest Ophthalmol Vis Sci. 2010;51 (2):744-751. doi:10.1167/iovs.09-3992

4. Abdelfattah NS, Amgad M, Salama AA, et al. Development of an Arabic version of the National Eye Institute Visual Function Questionnaire as a tool to study eye diseases patients in Egypt. Int $J$ Ophthalmol. 2014;7(5):891. doi:10.3980/j.issn.2222-3959.2 014.05.27

5. Mangione CM, Lee PP, Gutierrez PR, Spritzer K, Berry S, Hays RD. Development of the 25-list-item national eye institute visual function questionnaire. Arch Ophthalmol. 2001;119(7):1050-1058. doi:10.1001/archopht.119.7.1050

6. Schulze-Bonsel K, Feltgen N, Burau H, Hansen L, Bach M. Visual acuities "hand motion" and "counting fingers" can be quantified with the Freiburg visual acuity test. Invest Ophthalmol Vis Sci. 2006;47 (3):1236-1240. doi:10.1167/iovs.05-0981

7. Li M, Gong L, Chapin WJ, Zhu M. Assessment of vision-related quality of life in dry eye patients. Invest Ophthalmol Vis Sci. 2012;53(9):5722-5727. doi:10.1167/iovs.11-9094

8. Papageorgiou E, Hardiess G, Schaeffel F, et al. Assessment of vision-related quality of life in patients with homonymous visual field defects. Graefes Arch Clin Exp Ophthalmol. 2007;245 (12):1749-1758. doi:10.1007/s00417-007-0644-z
9. Murat Uyar O, Kapran Z, Akkan F, Cilsim S, Eltutar K. Vitreoretinal surgery for retained lens fragments after phacoemulsification. Eur J Ophthalmol. 2003;13(1):69-73. doi:10.1177/112067210301300110

10. Koh KM, Kim HS, Cho HJ, et al. Surgical outcomes of 23-gauge vitrectomy for the management of lens fragments dropped into the vitreous cavity during cataract surgery. Saudi J Ophthalmol. 2014;28 (4):253-256. doi:10.1016/j.sjopt.2014.01.001

11. Hutton WL, Snyder WB, Aiser AV. Management of surgically dislocated intravitreal lens fragments by pars plana vitrectomy. Ophthalmology. 1978;85(2):176-189. doi:10.1016/S0161-6420(78)35685-2

12. Margherio RR, Margherio AR, Pendergast SD, et al. Vitrectomy for retained lens fragments after phacoemulsification. Ophthalmology. 1997;104(9):1426-1432. doi:10.1016/S0161-6420(97)30120-1

13. Merani R, Hunyor AP, Playfair TJ, et al. Pars plana vitrectomy for the management of retained lens material after cataract surgery. $\mathrm{Am}$ J Ophthalmol. 2007;144(3):364-370. doi:10.1016/j.ajo.2007.05.027

14. Gilliland GD, Hutton WL, Fuller DG. Retained intravitreal lens fragments after cataract surgery. Ophthalmology. 1992;99 (8):1263-1269. doi:10.1016/S0161-6420(92)31814-7

15. Ng JQ, Morlet N, Pearman JW, et al. Management and outcomes of postoperative endophthalmitis since the endophthalmitis vitrectomy study: the Endophthalmitis Population Study of Western Australia (EPSWA)'s fifth report. Ophthalmology. 2005;112(7):1199-1206. doi:10.1016/j.ophtha.2005.01.050

16. Meleth AD, Carvounis PE. Outcomes of vitrectomy for tractional retinal detachment in diabetic retinopathy. Int Ophthalmol Clin. 2014;54(2):127-139. doi:10.1097/IIO.0000000000000021

17. Thompson JT, de Bustros S, Michels RG, Rice TA. Results and prognostic factors in vitrectomy for diabetic traction retinal detachment of the macula. Arch Ophthalmol. 1987;105(4):497-502. doi:10.1001/archopht.1987.01060040067035

18. Schoenberger SD, Miller DM, Riemann CD, et al. Outcomes of 25 -gauge pars plana vitrectomy in the surgical management of proliferative diabetic retinopathy. Ophthalmic Surg Lasers Imaging Retina. 2011;42(6):474-480. doi:10.3928/15428877-20110901-02

19. Yorston D, Wickham L, Benson S, Bunce C, Sheard R, Charteris D. Predictive clinical features and outcomes of vitrectomy for proliferative diabetic retinopathy. $B r J$ Ophthalmol. 2008;92(3):365-368. doi:10.1136/bjo.2007.124495

20. Rice TA, Michels RG, Rice EF. Vitrectomy for diabetic traction retinal detachment involving the macula. Am J Ophthalmol. 1983;95(1):22-33. doi:10.1016/0002-9394(83)90330-6

21. Tolentino FI, Freeman HM, Tolentino FL. Closed vitrectomy in the management of diabetic traction retinal detachment. Ophthalmology. 1980;87(11):1078-1089. doi:10.1016/S0161-6420(80)35115-4

22. Williams DF, Williams GA, Hartz A, Mieler WF, Abrams GW, Aaberg TM. Results of vitrectomy for diabetic traction retinal detachments using the en bloc excision technique. Ophthalmology. 1989;96 (6):752-758. doi:10.1016/S0161-6420(89)32813-2

23. Michels RG. Vitrectomy for complications of diabetic retinopathy. Arch Ophthalmol. 1978;96(2):237-246. doi:10.1001/ archopht.1978.03910050105001

24. Mason JO III, Colagross CT, Haleman T, et al. Visual outcome and risk factors for light perception and no light perception vision after vitrectomy for diabetic retinopathy. Am J Ophthalmol. 2005;140 (2):231-e1. doi:10.1016/j.ajo.2005.02.052

25. Margrain TH, Nollett C, Shearn J, et al. The Depression in Visual Impairment Trial (DEPVIT): trial design and protocol. $B M C$ Psychiatry. 2012;12(1):1-8. doi:10.1186/1471-244X-12-57

26. Du Y, Mo XH, Li XL, Zeng J, Luo W, Huang ML. Vision-related quality of life and depression in rhegmatogenous retinal detachment patients. Medicine. 2019;98(4):e14225. doi:10.1097/MD.0000000000014225

27. Brenner MH, Curbow B, Javitt JC, Legro MW, Sommer A. Vision change and quality of life in the elderly: response to cataract surgery and treatment of other chronic ocular conditions. Arch Ophthalmol. 1993;111(5):680-685. doi:10.1001/archopht.1993.01090050114040 
28. Ishii K, Kabata T, Oshika T. The impact of cataract surgery on cognitive impairment and depressive mental status in elderly patients. Am J Ophthalmol. 2008;146(3):404-409. doi:10.1016/j. ajo.2008.05.014
29. Brydges CR. Effect size guidelines, sample size calculations, and statistical power in gerontology. Innov Aging. 2019;3(4):igz036. doi:10.1093/geroni/igz036

\section{Publish your work in this journal}

Clinical Ophthalmology is an international, peer-reviewed journal covering all subspecialties within ophthalmology. Key topics include: Optometry; Visual science; Pharmacology and drug therapy in eye diseases; Basic Sciences; Primary and Secondary eye care; Patient Safety and Quality of Care Improvements. This journal is indexed on PubMed

Submit your manuscript here: https://www.dovepress.com/clinical-ophthalmology-journal
Central and CAS, and is the official journal of The Society of Clinical Ophthalmology (SCO). The manuscript management system is completely online and includes a very quick and fair peer-review system, which is all easy to use. Visit http://www.dovepress.com/ testimonials.php to read real quotes from published authors. 